Published by Cambridge University Press

This is an Open Access article, distributed under the terms of the Creative Commons

Attribution-NonCommercial-ShareAlike licence (http://creativecommons.org/licenses/by-nc-sa/4.0/),

which permits non-commercial re-use, distribution, and reproduction in any medium, provided the same

Creative Commons licence is included and the original work is properly cited. The written permission of

Cambridge University Press must be obtained for commercial re-use.

doi:10.1017/S0022377820000203

\title{
Accelerated methods for direct computation of fusion alpha particle losses within, stellarator optimization
}

\author{
Christopher G. Albert ${ }^{\oplus 1,2,} \dagger$, Sergei V. Kasilov ${ }^{2,3}$ and Winfried Kernbichler ${ }^{2}$ \\ ${ }^{1}$ Max-Planck-Institut für Plasmaphysik, 85748 Garching, Germany \\ ${ }^{2}$ Fusion@ÖAW, Institute of Theoretical and Computational Physics, Graz University of Technology, \\ 8010 Graz, Austria \\ ${ }^{3}$ Institute of Plasma Physics, National Science Center 'Kharkov Institute of Physics and Technology', \\ 61108 Kharkov, Ukraine
}

(Received 27 December 2019; revised 4 March 2020; accepted 4 March 2020)

Accelerated statistical computation of collisionless fusion alpha particle losses in stellarator configurations is presented based on direct guiding-centre orbit tracing. The approach relies on the combination of recently developed symplectic integrators in canonicalized magnetic flux coordinates and early classification into regular and chaotic orbit types. Only chaotic orbits have to be traced up to the end, as their behaviour is unpredictable. An implementation of this technique is provided in the code SIMPLE (symplectic integration methods for particle loss estimation, Albert et al., 2020b, doi:10.5281/zenodo.3666820). Reliable results were obtained for an ensemble of 1000 orbits in a quasi-isodynamic, a quasi-helical and a quasi-axisymmetric configuration. Overall, a computational speed up of approximately one order of magnitude is achieved compared to direct integration via adaptive Runge-Kutta methods. This reduces run times to the range of typical magnetic equilibrium computations and makes direct alpha particle loss computation adequate for use within a stellarator optimization loop.

Key words: fusion plasma, plasma confinement, plasma simulation

\section{Introduction}

Finding a stellarator configuration with favourable properties for magnetic confinement fusion poses a high-dimensional multi-objective optimization problem. One of these objectives is to minimize the losses of energetic fusion alpha particles over their slowing-down time in order to be able to heat the bulk plasma of a reactor. Direct computation of such losses with usual numerical methods is relatively time consuming compared to other calculations within the optimization loop, in particular computation of three-dimensional (3-D) magnetohydrodynamic equilibria. This is why

$†$ Email address for correspondence: albert@alumni.tugraz.at 
fusion alpha loss estimation is often performed via faster proxy models (Nemov et al. 2005, 2008; Bader et al. 2019) that, however, cannot capture the full physics of drift orbits and, therefore, are suited only for the initial stage of optimization. The reason for this is the high alpha energy of $3.5 \mathrm{MeV}$, leading to the following consequences. On the one hand, alpha particles show a rather fast cross-field drift and rather wide guiding-centre orbits on the bounce time scale. On the other hand, the collisional decorrelation time of orbits from the magnetic field is very large because of very low collision frequencies for alphas. These two factors combined can lead to diverse and unpredictable behaviour of drift orbits in 3-D magnetic geometry. Therefore, direct tracing of an ensemble of orbits and statistical estimation of losses is still a preferable option for accurate results (Lotz et al. 1992; Nemov, Kasilov \& Kernbichler 2014).

To address this problem, a class of symplectic integrators for guiding-centre motion (Zhang et al. 2014) has recently been extended and applied (Albert, Kasilov \& Kernbichler 2020a) to alpha loss computation in stellarator configurations. Despite reaching a significant speed up of factor 3-6 compared to adaptive Runge-Kutta orbit integration, further improvements are required to reduce the wall-clock run time below the time needed to compute magnetohydrodynamic equilibria.

To reach this goal, fast symplectic guiding-centre integration is complemented by classification of orbits as regular or chaotic based on Poincaré maps. In this context, 'regular' orbits are those that stay bound to some surfaces, called 'drift surfaces' and, therefore, can never leave the confinement volume. In particular, most of passing particle orbits are regular, as their drift surfaces are close to magnetic surfaces and only somewhat corrugated by cross-field drifts. This fact has been used in the past to reduce the number of orbits that have to be traced (e.g. Nührenberg, Lotz \& Gori 1994), and has inspired the idea of using a more rigorous classification, as presented here. The method treats trapped and passing particles in the same way and, therefore, takes into account possible losses of particles which would be classified from their starting conditions as passing ('marginally passing particles'). It should be noted that in modern advanced stellarator concepts (Nührenberg \& Zille 1988; Mikhailov et al. 2002) not only most passing but also most trapped particles have regular orbits. Therefore, the gain in efficiency via classification is significant.

The combination of the two techniques allows us to reduce the wall-clock time for collisionless alpha loss estimation by another factor 2-5 compared to using symplectic schemes alone, and thus reaches the required target to be used directly in optimization. This claim is supported by the presented results for three stellarator reactor configurations of quasi-isodynamic (Drevlak et al. 2014), quasi-helical (Drevlak et al. 2018) and quasi-axisymmetric type (Henneberg et al. 2019), respectively. The implementation of the method is available in the code SIMPLE (Albert, Kasilov \& Kernbichler 2020b) as open source under the MIT License. To produce the present results, version 1.1.1 of SIMPLE has been used.

\section{Methods}

\subsection{Canonicalized flux coordinates}

The guiding-centre Lagrangian (Littlejohn 1983; Cary \& Brizard 2009) in magnetic flux coordinates, omitting the ignorable term with gyrophase velocity $\dot{\phi}$, is given by

$$
L_{\mathrm{gc}}=h_{r} \dot{r}+\left(m v_{\|} h_{\vartheta}+\frac{e}{c} A_{\vartheta}\right) \dot{\vartheta}+\left(m v_{\|} h_{\varphi}+\frac{e}{c} A_{\varphi}\right) \dot{\varphi}-H,
$$

where $h_{i}=B_{i} / B$ are covariant components of unit vectors in direction of the magnetic field $\boldsymbol{B}$, the triple $(r, \vartheta, \varphi)$ are some spatial straight field line magnetic 
flux coordinates, $m$ and $e$ are particle mass and charge, respectively, $c$ is the speed of light, $A_{k}=A_{k}(r)$ are covariant vector potential components and

$$
H=\frac{m v_{\|}^{2}}{2}+\mu B+e \Phi
$$

is the Hamiltonian containing magnetic moment $\mu$ and electrostatic potential $\Phi$.

In spatial coordinates with $h_{r}=0$ it is immediately possible to identify canonical momenta $p_{\vartheta}, p_{\varphi}$ of the reduced 4-D phase space in front of $\dot{\vartheta}$ and $\dot{\varphi}$ in $(2.1)$. An efficient means of transformation from arbitrary 3-D magnetic flux coordinates to such coordinates has been presented recently (Albert et al. 2020a), being a three-dimensional generalization and synthesis of Meiss \& Hazeltine (1990) and Li, Breizman \& Zheng (2016). Based on such a transformation, phase-space coordinates $z=\left(r, \vartheta, \varphi, p_{\varphi}\right)$ are used, with only $r$ remaining as a non-canonical variable, resulting in dependencies

$$
\begin{aligned}
& L_{\mathrm{gc}}(z, \dot{\vartheta}, \dot{\varphi})=p_{\vartheta}(z) \dot{\vartheta}+p_{\varphi} \dot{\varphi}-H(z), \\
& p_{\vartheta}(z)=m v_{\|}(z) h_{\vartheta}(r, \vartheta, \varphi)+\frac{e}{c} A_{\vartheta}(r), \\
& v_{\|}(z)=\frac{1}{m h_{\varphi}(r, \vartheta, \varphi)}\left(p_{\varphi}-\frac{e}{c} A_{\varphi}(r)\right) .
\end{aligned}
$$

Computations of the exact transformation in the treated stellarator equilibria show that $(r, \vartheta, \varphi)$ remain close to Boozer magnetic coordinates, becoming identical to them in the zero beta limit. It has been argued by Boozer (2005) that, in such coordinates, $h_{r}$ can be neglected for the purpose of orbit integration, thereby immediately providing approximate canonicalized flux coordinates. Using such coordinates would further increase performance, as they depend on fewer free 3-D parameters for which interpolants have to be computed during evaluation of the magnetic field. Even though for the current work the exact canonicalization of flux coordinates has been chosen, a comparison to computation in Boozer coordinates with neglected $h_{r}$ could be an interesting task for future investigations.

\subsection{Symplectic integration in non-canonical coordinates}

With canonical momenta given in terms of (partially) non-canonical coordinates, we can proceed to apply a generalized form of symplectic integration (Zhang et al. 2014; Albert et al. 2020a). Here, dependencies and derivatives of Hamiltonian $H$ are written in terms of non-canonical phase-space coordinates $z$, but the quadrature scheme relies on classical symplectic integrators in canonical coordinates (Hairer, Lubich \& Wanner 2006). For the present investigation, a semi-implicit symplectic Euler method has been employed. In step number $(n)$ with time difference $\Delta t$, two implicit equations

$$
\begin{aligned}
0 & =\frac{\partial p_{\vartheta}}{\partial r}\left(p_{\vartheta,(n+1)}-p_{\vartheta,(n)}\right)+\Delta t\left(\frac{\partial p_{\vartheta}}{\partial r} \frac{\partial H}{\partial \vartheta}-\frac{\partial p_{\vartheta}}{\partial \vartheta} \frac{\partial H}{\partial r}\right), \\
0 & =\frac{\partial p_{\vartheta}}{\partial r}\left(p_{\varphi,(n+1)}-p_{\varphi,(n)}\right)+\Delta t\left(\frac{\partial p_{\vartheta}}{\partial r} \frac{\partial H}{\partial \varphi}-\frac{\partial p_{\vartheta}}{\partial \varphi} \frac{\partial H}{\partial r}\right)
\end{aligned}
$$

are first solved in $r=r_{(n, \mathrm{ei})}$ and $p_{\varphi}=p_{\varphi,(n+1)}$. Partial derivatives of poloidal momentum $p_{\vartheta}$ and Hamiltonian $H$ are evaluated at $\left(r_{(n, \mathrm{ei})}, \vartheta_{(n)}, \varphi_{(n)}, p_{\varphi,(n+1)}\right)$, according to (2.2) 
and (2.4), expressing $v_{\|}$via (2.5). Subscripts $(n)$ denote values at current time $t$, and $(n+1)$ the ones at $t+\Delta t$. The internal stage $r_{(n, \text { ei })}$ (with subscript 'ei' for 'explicitimplicit' Euler) does not correspond to a full time step but remains sufficiently close to the actual radial position $r$ of the guiding centre. In particular, this evaluation point in phase space is also used to express the poloidal momentum at step $(n+1)$ via

$$
p_{\vartheta,(n+1)}=p_{\vartheta}\left(r_{(n, \mathrm{ei})}, \vartheta_{(n)}, \varphi_{(n)}, p_{\varphi,(n+1)}\right) .
$$

In the remaining stages, new positions $\vartheta, \varphi$ follow explicitly in two equations

$$
\begin{gathered}
\vartheta_{(n+1)}=\vartheta_{(n)}+\Delta t \frac{\partial H}{\partial r}\left(\frac{\partial p_{\vartheta}}{\partial r}\right)^{-1}, \\
\varphi_{(n+1)}=\varphi_{(n)}+\Delta t \frac{1}{h_{\varphi}}\left(v_{\|}-\frac{\partial H}{\partial r}\left(\frac{\partial p_{\vartheta}}{\partial r}\right)^{-1} h_{\vartheta}\right),
\end{gathered}
$$

where derivatives of $p_{\vartheta}$ and $H$, as well as $h_{\vartheta}$ and $v_{\|}$, are evaluated at the same phase point as in (2.6) and (2.7).

\subsection{Classification of Poincaré sections}

As discussed above, regular orbits are bound to 'drift surfaces' which reduce to a line (or set of closed lines) on some given cross-sections (Poincaré sections). For the present analysis and classification, we use two types of Poincaré section of the whole phase space defined by

$$
v_{\|}=0^{ \pm}
$$

with $v_{\|}$switching either from negative to positive $\left(0^{-}\right)$or vice versa $\left(0^{+}\right)$, and

$$
\varphi=\frac{2 \pi l}{N_{\text {per }}},
$$

where $N_{\text {per }}$ is the number of field periods of the configuration and $l$ is an integer between 0 and $N_{\text {per }}-1$. Each of these sections is illustrated based on orbits in figures 1-2.

The considered Poincaré sections are actually 3-D hypersurfaces in a 4-D phase space (ignoring the gyrophase and treating the conserved magnetic moment $\mu$ as an auxiliary parameter). Imposing the additional constraint of conservation of total energy $H$ together with one of (2.11)-(2.12), hypersurfaces are reduced to 1-D lines in the case of regular orbits, or structures of fractal dimension between one and two in the case of chaotic orbits. Classification of the kind of orbit at hand is performed with the help of the box-counting fractal dimension described below.

The box-counting fractal dimension of a set of points (see, e.g., Falconer 2014, chap. 3), also known as the Minkowski-Bouligand dimension, is defined as follows. One considers the number of boxes $N(\varepsilon)$ of width $\varepsilon$ required to cover all points in the set. In the limiting case of small boxes, the fractal dimension is obtained as a ratio of exponents via

$$
d_{f}=\lim _{\varepsilon \rightarrow 0} \frac{\log N(\varepsilon)}{\log \left(\varepsilon^{-1}\right)} .
$$

For a finite set of points and for numerical implementation, a sufficiently small finite minimum value of $\varepsilon$ is used instead of this limit. Figure 3 illustrates the behaviour in 
(a)

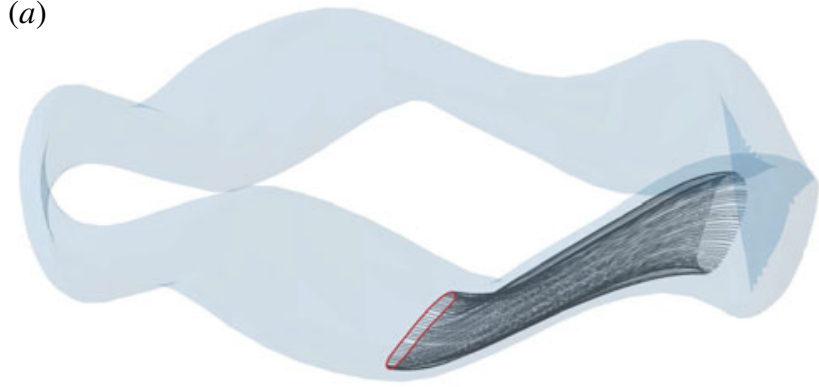

(b)

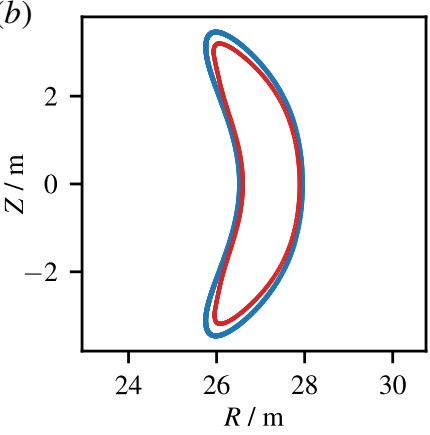

FIgURE 1. (a) Trapped orbit with a Poincaré section (red) at turning points $v_{\|}=0^{-}$, and outer plasma boundary (blue). Panel $(b)$ shows the projection of the section to the poloidal plane.
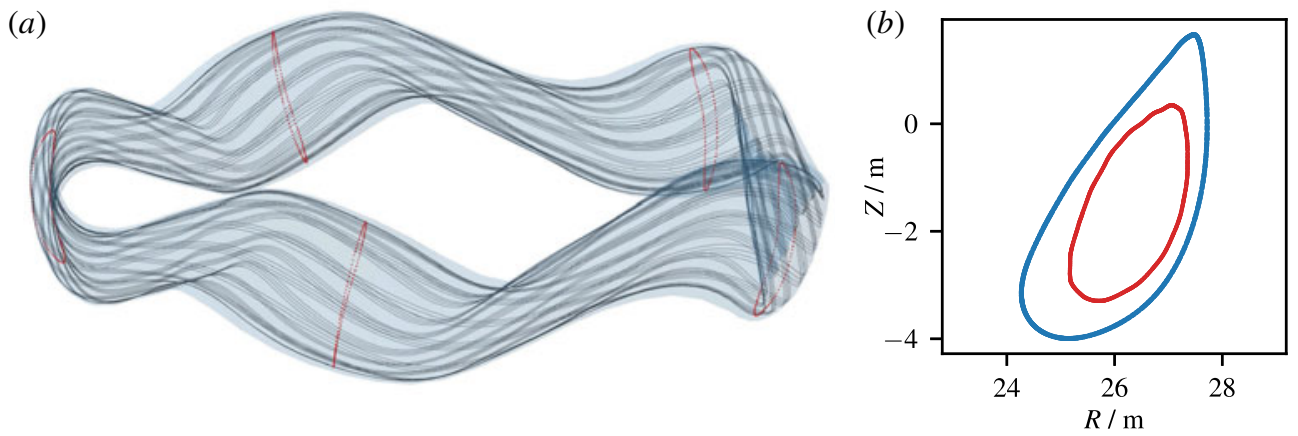

Figure 2. (a) Passing orbit with Poincaré sections (red) at toroidal field periods $\varphi=\varphi_{k}$, and outer plasma boundary (blue). (b) Projection of the section to the poloidal plane.

terms of a regular orbit and a chaotic orbit. In the regular case, the number $N(\varepsilon)$ grows linearly with $\varepsilon^{-1}$ and $d_{f}$ is computed close to one. In comparison, for the chaotic case, more boxes are required with smaller $\varepsilon$, leading to an estimated fractal dimension $d_{f}$ well between one and two. In the limit of points covering the whole section equally, $d_{f}$ approaches two.

In practice, to classify orbits, a threshold value for $d_{f}$ has to be set, below which orbits are classified as regular. In the present implementation this threshold has been determined empirically as $d_{f} \approx 1.6$ with adaptation to more conservative criteria with decreasing number of available points. If all section types (2.11)-(2.12) have a dimension below the threshold, the orbit is classified as regular. In the actual code realization it is more convenient to use, instead of the fractal dimension, a ratio $v$ of full boxes $N_{\text {full }}$ to the total number of boxes $N_{\text {box }}$, linked to $d_{f}$ by

$$
d_{f}=2 \frac{\log (v)}{\log \left(N_{\mathrm{box}}\right)}+2 .
$$

Here, the threshold is set directly as $v=0.2$.

Figure 4 shows an example of the estimated fractal dimension as a function of $N_{\text {box }}$ for a number of regular and chaotic orbits in a quasi-isodynamic stellarator configuration. Actual classification happens when the number of boxes becomes equal 

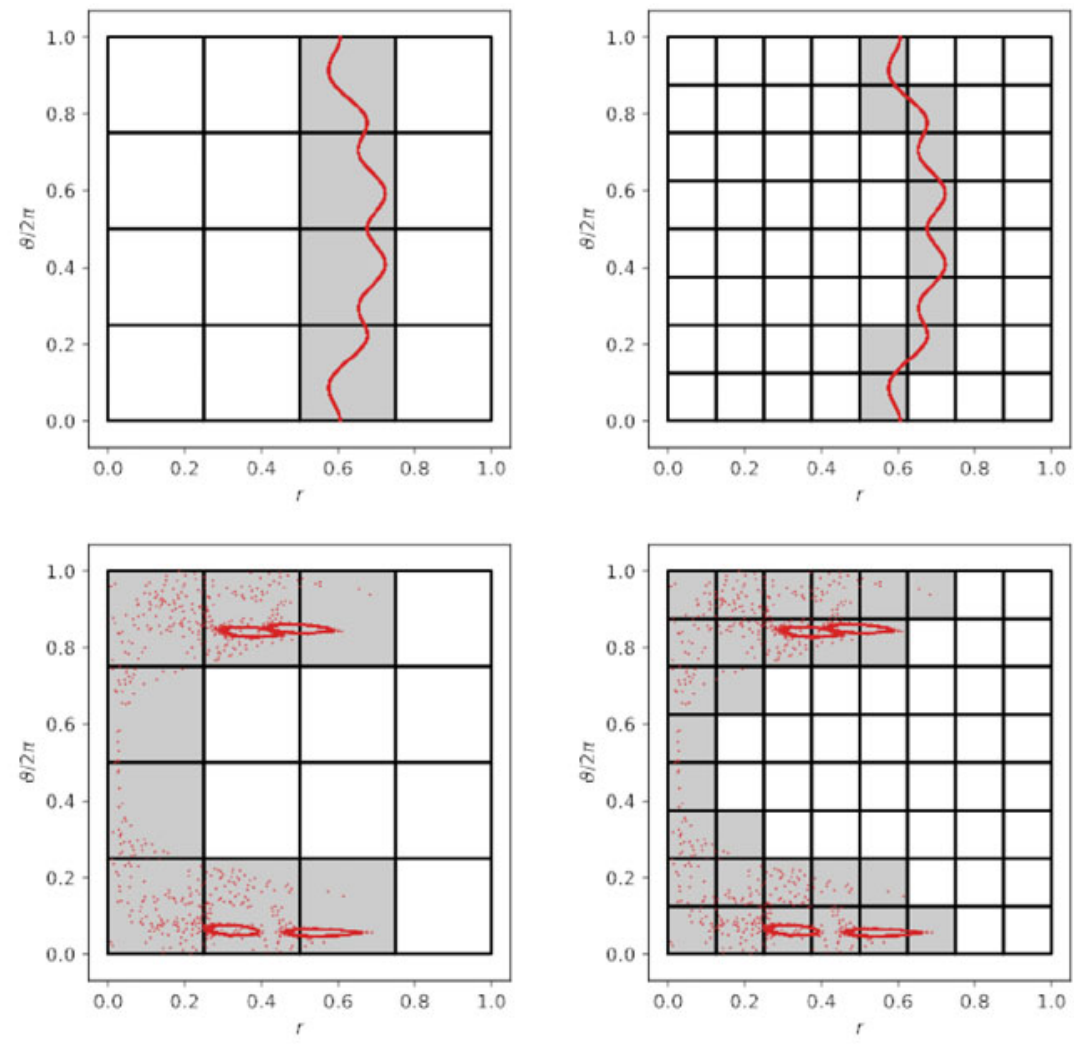

FIgURE 3. Classification of poloidal projections of Poincaré sections via box counting. The regular orbit in the upper plots has a one-dimensional projection, while the projection of the chaotic orbit on the bottom has a fractal dimension between one and two apparent on refinement.

to the number of orbit footprints in the Poincaré section. At higher $N_{\text {box }}$ this estimate can no longer be used, which is clearly seen from the behaviour of chaotic orbits.

\section{Results}

In this section numerical results on fusion alpha particle confinement are presented for three optimized stellarator configurations of similar (reactor) size with an on-axis magnetic field of $B_{0}=5 \mathrm{~T}$ : a quasi-isodynamic (QI) configuration (major radius $R=25 \mathrm{~m}$, aspect ratio $A=12$, plasma $\beta=4.9 \%$ ) of Drevlak et al. (2014), a quasi-helical $(\mathrm{QH})$ configuration $(R=19 \mathrm{~m}, A=8.7, \beta=3.9 \%)$ of Drevlak et al. (2018) and a quasi-axisymmetric (QA) configuration $(R=10.3 \mathrm{~m}, A=3.4, \beta=3.5 \%)$ of Henneberg et al. (2019). Resulting loss fractions over time match the ones in the references. It should be stressed once more that the high degree of optimization in these configurations makes the present classification method especially efficient, as most orbits are regular and losses are small. Still, the algorithm is expected to give a significant speed up in optimizing any magnetic configuration where the initial equilibrium already has an alpha loss fraction below 0.5 at the alpha particle slowing-down time $t_{\mathrm{s}}$. If this is not the case, alpha loss computations are anyway fast as long as losses happen well before $t_{\mathrm{s}}$ and tracing can be terminated frequently. 

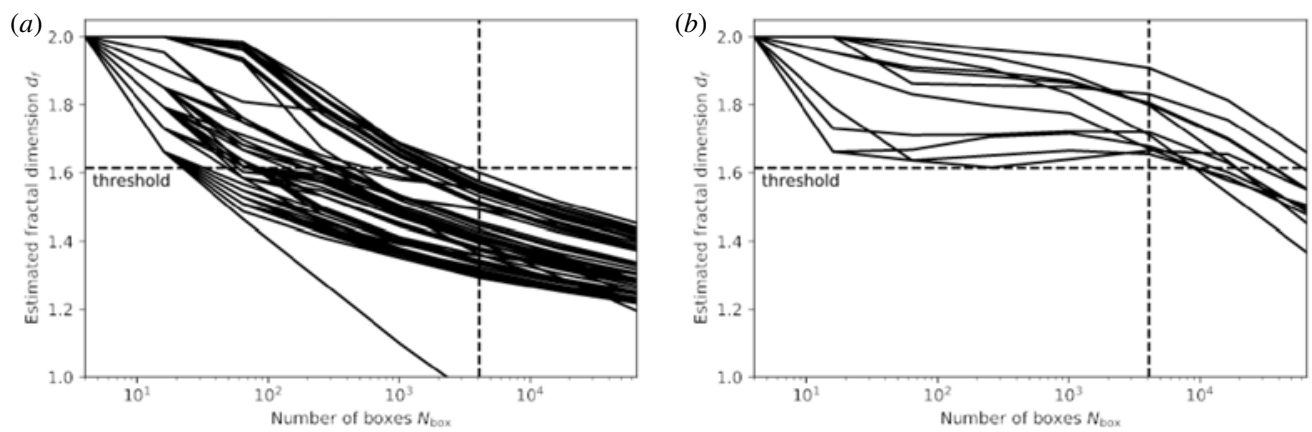

FIGURE 4 . Estimated fractal dimension $d_{f}$ by box counting versus number of boxes $N_{\text {box }}$ for several regular $(a)$ and chaotic $(b)$ orbits in a quasi-isodynamic configuration. Orbits are classified when $N_{\text {box }}$ equals the number of footprints using the threshold value $d_{f}=1.6$ (dashed lines).

Figures 5-7 show losses of alpha particles over time and a trapping parameter defined as

$$
\theta_{\text {trap }}=\left(\frac{\mu}{\mu_{\mathrm{tp}}}-1\right)\left(\frac{B_{\max }}{B_{\min }}-1\right)^{-1},
$$

where $\mu_{\mathrm{tp}}$ is the magnetic moment corresponding to the trapped passing boundary, and $B_{\min }$ and $B_{\max }$ are maximum and minimum values of the magnetic field modulus on the starting flux surface, respectively. For deeply trapped particles $\theta_{\text {trap }}=1$, while it vanishes at the trapped passing boundary. Negative values of $\theta_{\text {trap }}$ correspond to passing particles. In addition to black dots marking the actual loss of a test particle, shaded plots from a kernel density estimator based on the statistical samples have been added to mark regions of losses in time and phase space. Finally, the confined fraction $f_{c}$ over time is plotted as a curve.

Two cases are considered for each configuration where particles are started at a flux surface with radius $r=s \equiv \psi_{\text {tor }} / \psi_{\text {tor }}^{a}=0.6$ and $s=0.3$, respectively, where $\psi_{\text {tor }}$ is the toroidal magnetic flux and $\psi_{\text {tor }}^{a}$ its value at the outer plasma boundary. A total of $N_{\text {tot }}=1000$ randomly chosen orbits are traced up to physical time $t=1 \mathrm{~s}$ such that the slowing-down time is well covered. The estimated standard deviation $\sigma$ (random error) in the computed fraction $f_{c}$ of particles which remain confined scales inversely with this number as $\sigma=\sqrt{f_{c}\left(1-f_{c}\right) / N_{\text {tot }}}$. Classification of regular/chaotic orbit types is performed at $t=10^{-1} \mathrm{~s}$. After this point, regular orbits are considered to be confined and only chaotic orbits are traced further. Initial conditions are set to isotropic in velocity space with spatial positions distributed along the field line densely covering the flux surface so that particle density is constant in the flux tube. This method has worked without problems in the present cases and is also suitable in the general case where the coordinate system is not necessarily flux-surface aligned (Nemov et al. 2014). It has, however, some disadvantage if the starting surface is close to a low-order rational surface such that one needs a very long field line to cover it. In that case, the method of Bader et al. (2019) for flux coordinate systems would be preferable.

At the outer flux surface $s=0.6$ the QI configuration in figure 5 first shows some prompt losses $\left(t=10^{-4} \mathrm{~s}\right)$ near the trapped-passing boundary. Those are orbits such that they immediately cross the boundary at $s=1.0$ before even completing 

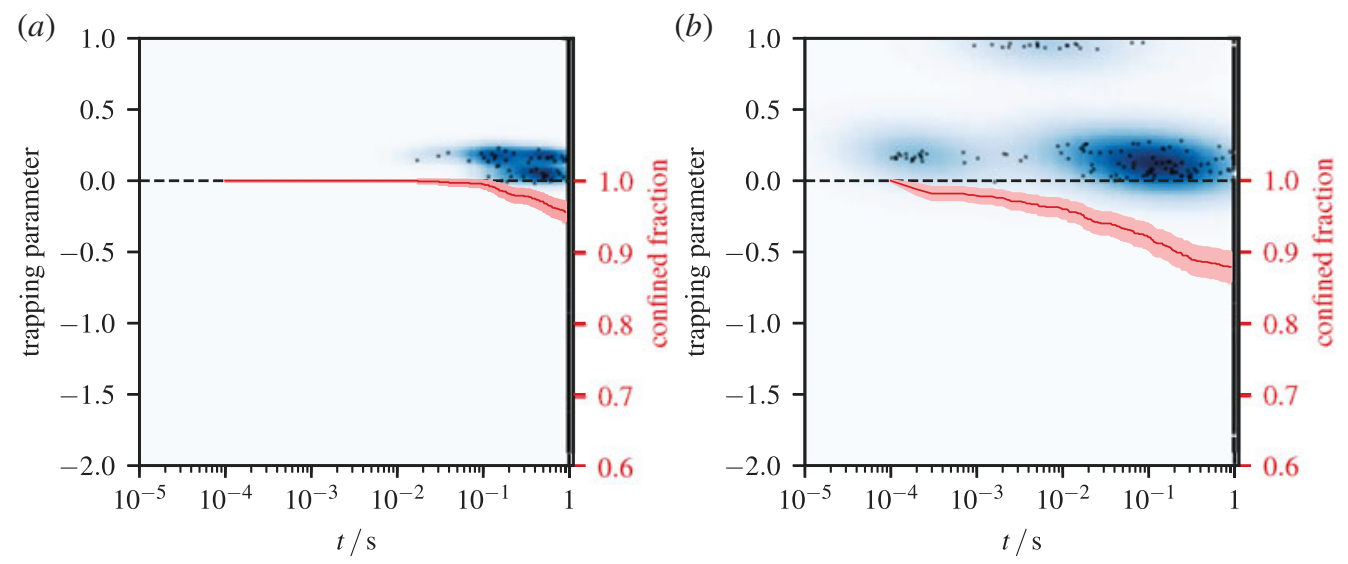

FIgURE 5. Alpha particle losses from $s=0.3(a)$ and $s=0.6(b)$ over time and trapping parameter (left axis) for a quasi-isodynamic stellarator configuration. Density plot over lost particles (black dots); confined fraction $f_{c}$ over time (lower curve, right axis). Error bands at $\pm 1.96 \sigma$ around this curve describe the $95 \%$ confidence interval due to the Monte Carlo error.
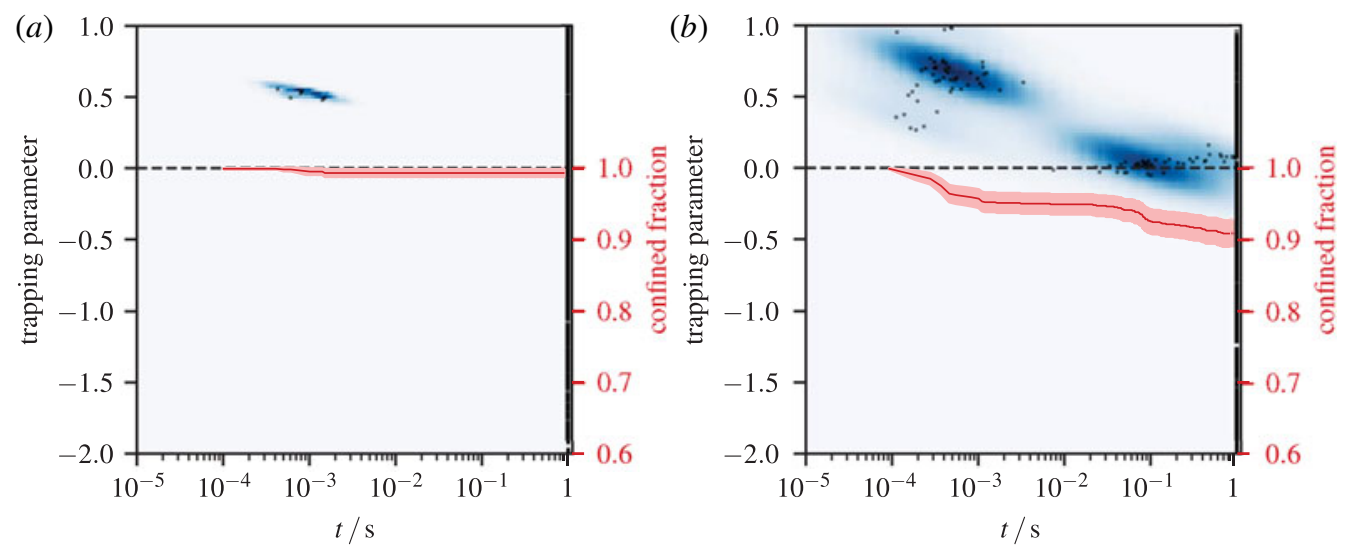

Figure 6. Alpha particle losses from $s=0.3(a)$ and $s=0.6(b)$ over time and trapping parameter (left axis) for a quasi-helical stellarator configuration in the style of figure 5 . Final losses at $s=0.3$ are below $2 \%$, including error bars.

a significant number of poloidal or toroidal turns in the device. At intermediate times between $t=10^{-3} \mathrm{~s}$ and $10^{-2} \mathrm{~s}$ originally deeply trapped particles are lost as drift motion and stochasticity remove them from their magnetic well. Finally, late losses at the trapped-passing boundary set in due to chaotic trajectories. In the QH configuration in figure 6 most losses from $s=0.6$ happen already early, between $t=10^{-4} \mathrm{~s}$ and $10^{-3} \mathrm{~s}$ for trapped particles that are neither deeply nor marginally trapped. Late losses are again located around the trapped-passing boundary, where chaotic effects are especially pronounced. The QA configuration in figure 7 spreads losses from $s=0.6$ more evenly over both time and trapping parameter. Most losses happen at $t$ between $10^{-3} \mathrm{~s}$ and $10^{-2} \mathrm{~s}$, depleting the population in the region near the trapped-passing boundary. 

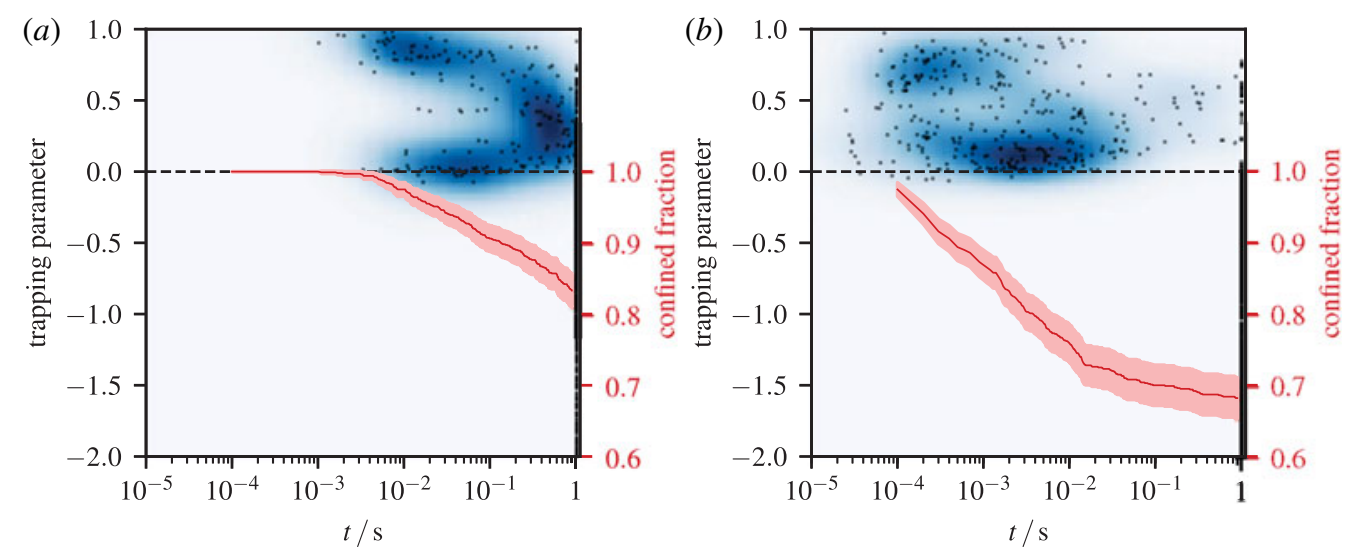

FIGURE 7. Alpha particle losses from $s=0.3(a)$ and $s=0.6(b)$ over time and trapping parameter (left axis) for a quasi-axisymmetric stellarator configuration in the style of figure 5 .

At the inner flux surface $s=0.3$ alpha confinement in the QI configuration in figure 5 is governed by late losses close to the trapped-passing boundary. In contrast, the $\mathrm{QH}$ configuration in figure 6 shows a few prompt losses and retains alphas up to the tracing time of $1 \mathrm{~s}$. In the quasi-axisymmetric configuration in figure 7 losses appear over the whole trapped region with deeply and marginally trapped orbits lost earlier. Here, the region of losses also extends into the marginally passing range.

If the threshold for the fractal dimension to identify chaotic orbits is set too high, the classification might produce 'false negatives'. In that case, an orbit is classified as regular despite being chaotic and lost if traced up to the end. In that case, final losses are underestimated if classification is used. In the computations for figures 5-7 no false negatives occurred, meaning that classification did not deteriorate results on late losses. In contrast, 'false positive' classification of chaotic orbits just increases computation time by tracing them up to the end, but does not influence the result. This trade-off between accuracy and computation time is adjusted via the choice of the threshold fractal dimension (2.13) to decide whether an orbit is chaotic. As mentioned above, this option has been left at default settings independent of the treated cases and could potentially be optimized.

Figure 8 shows the classification results depending on initial conditions in pitch parameter $v_{\|} / v$ and poloidal angle $\vartheta$ for $10^{4}$ orbits started at $s=0.6$ and $\varphi=\varphi_{n} / 2$, i.e. in the middle of the first field period. All three configurations show regular orbits in the passing region at some distance from the trapped-passing boundary. For the QI and QH configurations there is a clear separation of early losses of orbits that cross the plasma boundary soon and chaotic orbits that slowly diffuse away. Losses are localized at the trapped-passing boundary and in certain phase-space regions for more deeply trapped orbits. In the QA configuration most trapped orbits are chaotic, but some are lost relatively late due to slow stochastic diffusion. The described behaviour is also seen in figures 5-7. Therefore, the classification is of less use in the QA case, as most regular orbits are passing and could be identified in simpler ways. In contrast, for QI and QH types the method allows us to exclude also a large portion of the trapped region from further computation. A relatively small number of 'false positives' that are incorrectly classified as chaotic are seen, in particular in the QI configuration, and traced to the end despite being actually regular. 

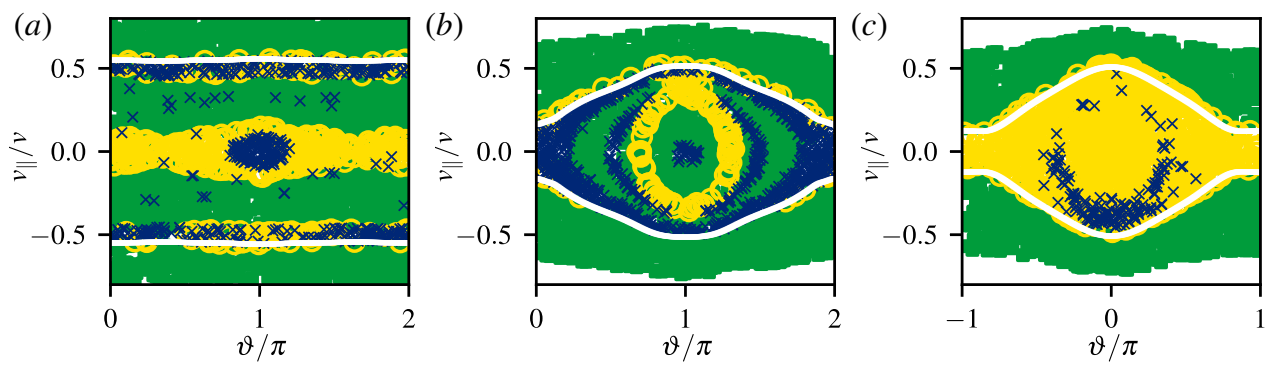

FIGURE 8. Orbit types over initial condition in $v_{\|} / v$ and $\vartheta$ from $s=0.6$ for QI $(a)$, QH (b) and QA configuration $(c)$. The background ( $(\boldsymbol{)})$ is filled by regular orbits, early losses before $t=0.1 \mathrm{~s}$ are marked as ' $O$ ', and chaotic orbits potentially causing late losses after $t=0.1 \mathrm{~s}$ as ' $\mathrm{X}$ ' with some 'false positives' visible that remain confined. The trappedpassing boundary is marked by a white line.

\begin{tabular}{|c|c|c|c|}
\hline Type & Flux surface $s$ & Regular trapped & Regular passing \\
\hline QI & 0.3 & 0.8219 & 0.9987 \\
\hline QI & 0.6 & 0.6751 & 0.9946 \\
\hline $\mathrm{QH}$ & 0.3 & 0.5343 & 0.9594 \\
\hline $\mathrm{QH}$ & 0.6 & 0.5884 & 0.9780 \\
\hline QA & 0.3 & 0.0927 & 0.9607 \\
\hline QA & 0.6 & 0.0657 & 0.9721 \\
\hline
\end{tabular}

TABLE 1. Fractions of regular orbits in the trapped and passing regions for different configurations.

Table 1 shows numerical values of respective regular fractions for trapped and passing orbits in the considered configurations. A significant portion of chaotic trapped orbits in the $\mathrm{QH}$ and $\mathrm{QA}$ configurations stays confined within $t=1 \mathrm{~s}$, which makes confinement properties of alphas acceptable even in cases where only a small fraction of trapped orbits are regular.

Computations were performed on the COBRA cluster of MPCDF on a single node with 40 cores/80 threads of Intel(R) Xeon(R) Gold 6126 CPUs. Table 2 shows a summary of computation wall-clock time using a symplectic integrator alone compared to added classification after $1 / 10$ th of the integration time. The obtained speed up of another factor 2-5 compared to an integration method that is already a factor 3-6 faster than conventional Runge-Kutta integration for alpha loss results of the same accuracy (Albert et al. 2020a) leads to a total speed up of factor 6-30 compared to conventional methods.

\section{Summary and outlook}

A combined method of symplectic integration and early classification has been presented to accelerate the computation of loss fractions of fusion alpha particles over their slowing-down time in stellarator reactor configurations. Reliable results could be obtained for three different stellarator configuration types within wall-clock times of approximately $10 \mathrm{~min}$. This corresponds to a speed up of approximately an order of magnitude compared to conventional methods and makes the technique 


\begin{tabular}{|c|c|c|c|}
\hline Type & Flux surface $s$ & No classification & With classification \\
\hline QI & 0.3 & $27: 26$ & $07: 28$ \\
\hline QI & 0.6 & $25: 21$ & $07: 25$ \\
\hline $\mathrm{QH}$ & 0.3 & $29: 33$ & $11: 39$ \\
\hline $\mathrm{QH}$ & 0.6 & $31: 26$ & $07: 24$ \\
\hline QA & 0.3 & $41: 53$ & $16: 55$ \\
\hline QA & 0.6 & $40: 03$ & $07: 23$ \\
\hline
\end{tabular}

TABLE 2. Wall-clock run times in minutes on $40 \mathrm{CPU}$ cores with hyperthreading for the considered configurations. Computation for the QA equilibrium at inner flux surfaces is least efficient, as most orbits are chaotic, even when confined over their slowing-down time.

useful for integrated in stellarator optimization, where computation times for magnetic equilibria are of the same order.

Currently, the approach is limited to collisionless orbits. Adding collisions could limit the effectiveness of early classification by additional diffusion and requires further investigations. Even though mis-classifications influencing final results were rare with default settings, the classification algorithm could benefit from additional tuning in order to become even more robust. Finally, the influence of using Boozer coordinates instead of exact canonicalized flux coordinates should be studied further, as it provides room for further efficiency improvements.

\section{Acknowledgements}

The authors would like to thank M. Heyn for pointing out the Minkowski dimension, M. Drevlak, S. Henneberg and F. Hindenlang for equilibrium configurations and validation, A. Boozer and A. Punjabi for useful comments and J. Ganglbauer for supporting investigations. This work has been carried out within the framework of the EUROfusion Consortium and has received funding from the Euratom research and training programmes 2014-2018 and 2019-2020 under grant agreement no. 633053. The views and opinions expressed herein do not necessarily reflect those of the European Commission. The study is a contribution to the Reduced Complexity Models grant number ZT-I-0010 funded by the Helmholtz Association of German Research Centers. Support from NAWI Graz, and from the OeAD under the WTZ grant agreement with Ukraine No. UA 04/2017 is gratefully acknowledged.

\section{REFERENCES}

Albert, C. G., Kasilov, S. V. \& Kernbichler, W. $2020 a$ Symplectic integration with noncanonical quadrature for guiding-center orbits in magnetic confinement devices. J. Comput. Phys. 403, 109065.

Albert, C. G., Kasilov, S. V. \& Kernbichler, W. $2020 \mathrm{~b}$ SIMPLE: Symplectic Integration Methods for Particle Loss Estimation v1.1.1, doi:10.5281/zenodo.3666820.

Bader, A., Drevlak, M., Anderson, D. T., Faber, B. J., Hegna, C. C., Likin, K. M., Schmitt, J. C. \& TAlmadge, J. N. 2019 Stellarator equilibria with reactor relevant energetic particle losses. J. Plasma Phys. 85 (5), 905850508.

Boozer, A. H. 2005 Physics of magnetically confined plasmas. Rev. Mod. Phys. 76 (4), 1071-1141.

CARY, J. R. \& Brizard, A. J. 2009 Hamiltonian theory of guiding-center motion. Rev. Mod. Phys. 81 (2), 693-738. 
Drevlak, M., Beidler, C. D., Geiger, J., Helander, P., Henneberg, S. A., Nührenberg, C. \& TURKIN, Y. 2018 New results in stellarator optimization. In 27th IAEA Fusion Energy Conf. IAEA Publications.

Drevlak, M., Beidler, C. D., Geiger, J., Helander, P. \& Turkin, Y. 2014 Quasi-isodynamic configuration with improved confinement. In Proc. EPS Conf. Plasma Phys., ECA, vol. 38F. European Physical Society.

Falconer, K. 2014 Fractal Geometry: Mathematical Foundations and Applications, 3rd edn. Wiley.

Hairer, E., Lubich, C. \& WANner, G. 2006 Geometric Numerical Integration: Structure-Preserving Algorithms for Ordinary Differential Equations. Springer.

Henneberg, S. A., Drevlak, M., Nührenberg, C., Beidler, C. D., Turkin, Y., Loizu, J. \& Helander, P. 2019 Properties of a new quasi-axisymmetric configuration. Nucl. Fusion 59 (2), 026014.

Li, M., Breizman, B. N. \& Zheng, L. 2016 Canonical straight field line magnetic flux coordinates for tokamaks. J. Comput. Phys. 326, 334-341.

Littlejohn, R. G. 1983 Variational principles of guiding centre motion. J. Plasma Phys. 29 (1), $111-125$

Lotz, W., Merkel, P., Nührenberg, J. \& Strumberger, E. 1992 Collisionless alpha-particle confinement in stellarators. Plasma Phys. Control. Fusion 34 (6), 1037-1052.

Meiss, J. D. \& Hazeltine, R. D. 1990 Canonical coordinates for guiding center particles. Phys. Fluids B 2 (11), 2563-2567.

Mikhailov, M. I., Shafranov, V. D., Subbotin, A. A., Isaev, M. Y., Nührenberg, J., Zille, R. \& COOPER, W. A. 2002 Improved particle confinement in stellarators with poloidally closed contours of the magnetic field strength. Nucl. Fusion 42 (11), L23-L26.

Nemov, V. V., Kasilov, S. V. \& KernbiChleR, W. 2014 Collisionless high energy particle losses in optimized stellarators calculated in real-space coordinates. Phys. Plasmas 21 (6), 062501.

Nemov, V. V., Kasilov, S. V., Kernbichler, W. \& Leitold, G. O. 2005 The $\nabla B$ drift velocity of trapped particles in stellarators. Phys. Plasmas 12, 112507.

Nemov, V. V., Kasilov, S. V., Kernbichler, W. \& Leitold, G. O. 2008 Poloidal motion of trapped particle orbits in real-space coordinates. Phys. Plasmas 15, 052501.

NÜHrenberg, J., LotZ, W. \& GoRI, S. 1994 Quasi-axisymmetric tokamaks. In Theory Fusion Plasmas Proc. Jt. Varenna-Lausanne Int. Work (ed. E. Sindoni, F. Troyon \& J. Vaclavic), pp. 3-12. Editrice Compositori.

NÜHREnBERG, J. \& ZILle, R. 1988 Quasi-helically symmetric toroidal stellarators. Phys. Lett. A 129 (2), 113-117.

Zhang, R., LiU, J., TAng, Y., Qin, H., XiaO, J. \& ZhU, B. 2014 Canonicalization and symplectic simulation of the gyrocenter dynamics in time-independent magnetic fields. Phys. Plasmas 21 (3), 32504 . 